\title{
Factores que influyen en el coeficiente de estabilidad: Diámetro y longitud
}

\section{Factors influencing stability. Diameter and length}

\author{
CERVANTES HARO N* \\ ENCABO DURÁN MJ** \\ CALDERÍN PÉREZ S*** \\ ARAGONESES LAMMAS JM****
}

\begin{abstract}
Cervantes Haro N, Encabo Durán MJ, Calderín Pérez S, Aragoneses Lamas JM. Factores que influyen en el coeficiente de estabilidad: Diámetro y longitud. Av Periodon Implantol. 2014; 26, 1: 39-44.
\end{abstract}

\section{RESUIMEN}

Objetivos: Determinar si el diámetro y la longitud de los implantes dentales son factores determinantes de la estabilidad primaria.

Material y métodos: Se colocaron 17 implantes unitarios OIN de la casa Osseolife $₫$ Implant System de diámetros 3,75, 4,25 y 5,0 $\mathrm{mm}$ y longitudes de 10 y $11,5 \mathrm{~mm}$. El coeficiente de estabilidad fue medido mediante el Osstell Mentor ${ }^{\circledR}$ durante la cirugía de colocación. Los resultados obtenidos fueron comparados en cuanto al diámetro y la longitud con el estadístico para muestras independientes $t$ de Student.

Resultados: El coeficiente de estabilidad mayor fue para los implantes de diámetro estrecho (3,75 $\mathrm{mm}$ ) y cortos $(10 \mathrm{~mm})$ con un ISQ de 75,5 y 76,0 respectivamente. Para los otros diámetros y longitud los resultados fueron: Para los implantes de $4,25 \mathrm{~mm}$ y $5,0 \mathrm{~mm}$ obtuvieron un ISQ de 74,7, y 74,33 , respectivamente, y para el implante denominado largo con longitud de $11,5 \mathrm{~mm}$ el coeficiente de estabilidad fue de 70,85. En ambos parámetros, objeto de estudio, no se encontraron diferencias estadísticamente significativas con $\mathrm{p}>0,05$.

Conclusiones: Tanto la longitud como el diámetro no son factores determinantes del coeficiente de estabilidad primaria.

PALABRAS CLAVE: Implantes dentales, estabilidad, diámetro, longitud.

\section{SUMMARY}

Objectives: To determine if the diameter and length of the dental implants aredeterminants of primary stability.

Materials and methods: 17 unitary OIN Osseolife ${ }^{\circledR}$ Implant System implants were placed with diameters of $3.75,4.25$ and $5.0 \mathrm{~mm}$ and lengths of 10 and $11.5 \mathrm{~mm}$. The stability coefficient was measured using the Mentor ${ }^{\circledR}$ Osstell placement during surgery. The results were compared in terms of diameter and length with the statistic for independent samples $t$ test.

Results: The stability coefficient was highest for small diameter implants $(3.75 \mathrm{~mm})$ and short ones $(10 \mathrm{~mm})$ with a ISQ of 75.5 and 76.00 respectively. For other diameters and length results were: implants of $4.25 \mathrm{~mm}$ and $5.0 \mathrm{~mm}$ obtained a ISQ of 74.7 , and 74.33 respectively and for the implant

\footnotetext{
* Doctor en Odontología. Profesora del Máster de Cirugía, Implantología y Periodoncia de la Universidad de Alcalá y la Institución Universitaria Mississippi.

** Profesora del Máster de Cirugía, Implantología y Periodoncia de la Universidad de Alcalá y la Institución Mississippi.

*** Alumna del Máster de Cirugía, Implantología y Periodoncia de la Universidad de Alcalá y la Institución Mississippi.

**** Doctor en Odontología. Director del Máster de Cirugía, Implantología y Periodoncia de la Universidad de Alcalá y la Institución Universitaria Mississippi. Director de área de la Universidad Europea de Madrid.
} 
called long (11.5 mm length) stability coefficient was 70.85 . In both parameters under study, there were no statistically significant differences with $\mathrm{p}>0.05$.

Conclusions: Both the length and the diameter are not determinants of primary stability coefficient.

KEY WORDS: Dental implants, stability, diameter, length.

Fecha de recepción: 28 de enero de 2013.

Fecha de aceptación: 29 de abril de 2013.

\section{INTRODUCCIÓN}

La pérdida de un diente puede producir en algunos pacientes problemas, del tipo funcionales como la dificultad para masticar o psicológicos debido a las alteraciones estéticas (1). La rehabilitación oral con implantes dentales un tratamiento para la sustitución de los dientes ausentes, desde hace más de dos décadas (2). Los implantes dentales en sus inicios fueron un fracaso debido a la no biocompatibilidad de los materiales, hasta que en 1965 Brånemark consiguió la osteointegración del titanio al hueso (3). La técnica descrita por Brånemark ha sido utilizada como procedimiento estandarizado desde 1971. El implante original era de $10 \mathrm{~mm}$ de longitud y de $3,75 \mathrm{~mm}$ de diámetro, con un diseño similar al que se utiliza en la actualidad (4). El protocolo propuesto por Brånemark en 1969 era una técnica quirúrgica en dos fases, donde el implante era colocado en el hueso y posteriormente cubierto por la mucosa oral, durante 6 meses en maxilar superior y 4 meses en maxilar inferior, evitando así que los implantes sean cargados funcionalmente o tengan posibilidades de ser contaminados (1). Aunque este protocolo sigue siendo el más generalizado, en la actualidad se utiliza también el protocolo denominado carga inmediata (5), permitiendo a los clínicos acortar los tiempos de tratamiento, mejorando así la calidad de vida del paciente, no sólo en la función masticatoria sino también la estética $(6,7)$.

Una de la principales preocupaciones del clínico es saber en qué momento del tratamiento se puede conectar el implante al pilar protésico para la rehabilitación protésica, que se puede determinar mediante la estabilidad del implante. Podemos definir la estabilidad primaria como la unión mecánica entre hueso e implante en el momento de la colocación de éste (8, 9); y estabilidad secundaria es la que se produce una vez finalizado el proceso de osteointegración (10). Para medir la estabilidad se han utilizado diferentes métodos, como el torque de inserción $(11,12)$, métodos radiográficos (12), análisis histológicos (13), Periotest ${ }^{\circledR}$ (14), entre otros. Todos estos métodos tienen diferentes limitaciones, bien por ser técnicas agresivas para los pacientes, bien por no ser reproducibles o simplemente estar relegados al ámbito del laboratorio experimental. Durante años se han ido investigando otros métodos de estudio hasta que Meredith, en 1996, $(15,16)$ describió el Análisis de la Frecuencia de la Resonancia (RFA). Este método permite estudiar la estabilidad del implante dental en diferentes momentos de la cicatrización ósea, siendo los resultados obtenidos reproducibles intraoperador e interoperador. Este método de estudio está basado en el análisis de la frecuencia, que consta de un transductor en forma de L (1) que va atornillado al implante perpendicular a la cresta ósea (17). Este transductor es estimulado mediante una señal sinusoide con un frecuencia de 5 a $10 \mathrm{KHz} .(1,15,18)$, siendo esta señal traducida mediante el análisis de la frecuencia. Los resultados obtenidos son representados por el coeficiente de estabilidad del implante (ISQ) con un intervalo de movilidad del implante de 0 a 100, siendo 100 el de mayor estabilidad $(9,19,20)$.

En la actualidad, se ha desarrollado el Osstell Mentor ${ }^{\circledR}$ que a diferencia del RFA, el transductor no está unido mediante cable, si no que éste, emite una señal electromagnética a un dispositivo atornillado al implante de forma manual, que se denomina Smartpeg ${ }^{\circledR}(21)$.

El objetivo de este estudio es determinar si la longitud y el diámetro son factores determinantes del coeficiente de estabilidad primaria de los implantes dentales.

\section{MATERIAL Y MÉTODOS}

El presente estudio fue desarrollado en el Máster de Implantología, Periodoncia y Cirugía de la Universidad de Alcalá impartido en la Institución Universitaria Mississippi.

\section{Selección del paciente}

Este estudio se llevó a cabo en 17 pacientes con ausencia de un diente, de los cuales 10 fueron mujeres y 
7 hombres entre 30 y 70 años. A todos los pacientes se les entregó consentimiento informado sobre el estudio para que lo firmaran, se les hizo ortopantomografía para una primera valoración y terapia periodontal básica a todos aquellos pacientes que lo necesitaron con instrucciones de higiene.

\section{Criterios de inclusión}

- Pacientes mayores de edad.

- Ausencia de patología sistémica.

- Cantidad y calidad ósea adecuada para buena estabilidad primaria.

- Pacientes periodontalmente estables.

- Pacientes no fumadores.

- Pacientes ASA tipos I y II.

- Relación corona implante no mayor de 1:1.

\section{Criterios de exclusión}

- Expectativas no realistas.

- Enfermedades autoinmunes, psiquiátricas.

- Pacientes portadores de sobredentaduras.

- Pacientes con extracciones realizadas 3 meses o menos anterior a la cirugía.

\section{Procedimiento quirúrgico}

A todos los pacientes se les prescribió profilaxis antibiótica, $2 \mathrm{~g}$ de amoxicilina (600 $\mathrm{mg}$ de clindamicina a alérgicos a la penicilina) 1 hora antes de la cirugía. Los implantes utilizados fueron los OIN de Osseolife $\AA$ Implant System de 3,75, 4,25 y 5 mm de diámetro y longitudes de 10 y $11,5 \mathrm{~mm}$. La osteotomía y colocación de los implantes se hizo según el protocolo marcado por la empresa comercial. Una vez insertado el implante en su totalidad en el lecho óseo, se procede a la medida del coeficiente de estabilidad. Una vez medido se coloca el tornillo de cierre y se suturó con seda de 3/0 de la casa Aragó $\circledast$. En cuanto al tratamiento posquirúrgico, se les recetó amoxicilina de $750 \mathrm{mg}$ o clindamina de $300 \mathrm{mg}, 3$ tomas diarias durante una semana e Ibuprofeno $600 \mathrm{mg}$ para el controlar el dolor y la inflamación. Además se les explicó las normas postquirúrgicas y técnicas de higiene. A siete días de la cirugía, se citó a los pacientes para la retirada de la sutura.

\section{Obtención de los datos}

La recogida se hizo una vez colocado el implante en la osteotomía y justo antes de la colocación del tornillo de cierre. Para registrar el coeficiente de estabilidad primaria, se atornilla el smartpeg $®$ al implante de forma manual con ayuda del transportador con una fuerza de $10 \mathrm{~N}$, que es la recomendada por la empresa Osstell@. Una ver atornillado el elemento se toma el Osstell Mentor ${ }^{\circledR}$ con la antena perpendicular al Smartpeg ${ }^{\circledR}$ y desde las cuatro orientaciones posibles, vestibular, lingual, mesial y distal, se espera a que este emita un pitido continuo, que indica que la lectura ha finalizado, apareciendo el resultado en la pantalla led del Osstell Mentor ${ }^{\circledR}$. Una vez finalizada la medida, se retira el smartpeg del implante con el transportador.

\section{ANÁLISIS ESTADÍSTICO}

Para el análisis estadístico se ha utilizado el Software SPSS $\circledast$ y para el estudio comparativo se aplicó la prueba $t$ de Student para muestras independientes con un nivel de significancia del $95 \%$.

\section{RESULTADOS}

Un total de 17 implantes fueron colocados de los cuales, cuatro eran implante estrecho de $3,75 \mathrm{~mm}(\mathrm{Oe})$, nueve implantes medianos de $4,25 \mathrm{~mm}(\mathrm{Om})$ y tres implantes anchos de $5 \mathrm{~mm}(\mathrm{Oa})$ de diámetro. En cuanto a la distribución según la longitud, 10 implantes tenía $10 \mathrm{~mm}$ (Oc) y 7 implantes tenían una longitud de $11,5 \mathrm{~mm}(\mathrm{Ol})$. Los resultados obtenidos en este estudio según el diámetro son los siguientes: para los implantes $\mathrm{Oe}, \mathrm{Om}, \mathrm{Oa}$, se obtuvo un coeficiente de estabilidad de media, 75,5, 74,7, 74,33 ISQ, respectivamente y, si se refiere a la longitud, la media obtenida para los implantes Oc, Ol es de 76,70 y 70,85 ISQ, respectivamente, siendo el implante corto de $10 \mathrm{~mm}$ el que consiguió mayor coeficiente de estabilidad (Figura 1). La desviación típica de las medias se muestra en la Tabla 1.

Al comparar los diámetros entre sí, no se han obtenido diferencias estadísticamente significativas siendo p>0,05 y en el grupo de estudio donde se compararon las dos longitudes de implantes, tampoco se han encontrado diferencias estadísticamente significativas con $\mathrm{p}=0,116$.

\section{DISCUSIÓN}

Desde que se comenzó con la implantología dental como actualmente se conoce, descrita por Brånemark en $1967(22,23)$, se ha intentado predecir el éxito de los implantes. En la actualidad, debido a las exigen- 


\section{TABLA 1.- RESULTADOS OBTENIDOS EN LOS DIFERENTES GRUPOS DE ESTUDIO}

\begin{tabular}{|l|c|c|c|c|c|c|}
\hline & Media & $\begin{array}{c}\text { Límite } \\
\text { inferior }\end{array}$ & $\begin{array}{c}\text { Límite } \\
\text { superior }\end{array}$ & $\begin{array}{c}\text { Desviación } \\
\text { típica }\end{array}$ & $\begin{array}{c}\text { Error } \\
\text { típico }\end{array}$ & Mediana \\
\hline Oe & 75,5000 & 65,3591 & 83,6409 & 5,0745 & 2,8723 & 73,0000 \\
Om & 74,7000 & 68,2217 & 81,1783 & 9,0560 & 2,8638 & 76,0000 \\
Oa & 74,3333 & 55,6885 & 92,9782 & 7,5055 & 4,3333 & 74,0000 \\
Oc & 76,7000 & 72,6667 & 80,7333 & 5,6382 & 1,7829 & 77,0000 \\
Ol & 70,8571 & 62,6468 & 79,0674 & 8,8775 & 3,3554 & 72,0000 \\
\hline
\end{tabular}

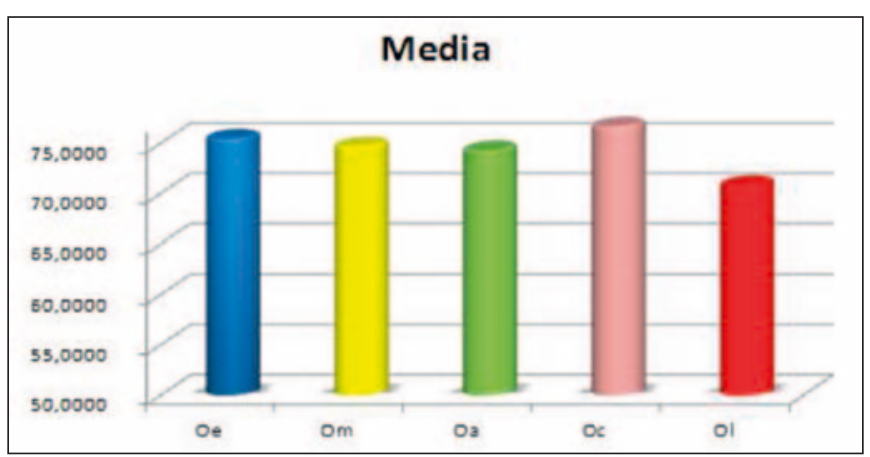

Fig. 1: Diagrama de barras representativo de las medias en unidades ISQ.

cias tanto de profesionales como de los pacientes, los tiempos de tratamiento se han ido acortando. La necesidad de saber el estado del implante en relación con el hueso ha dado lugar a la búsqueda de métodos para su valoración. Además, nos permiten saber si un implante es apto para ser cargado en el momento de la cirugía (24), ya que cada vez es más común realizar carga inmediata, quedando el protocolo descrito por Brånemark relegado a casos en los que hay grandes defectos óseos o necesidad de regeneración de tejidos blandos.

La razón de este estudio es conocer qué características de los implantes afectan a la estabilidad del implante, como puede ser la longitud y el diámetro.

La necesidad de medir la estabilidad del implante hicieron que se compararan distintos tipos de métodos, como Yoshihiro Ito y cols. (12) en 2008, que compararon tres métodos de estudio, Torque de inserción, Periotest $₫$ y RFA. También es importante a la hora de utilizar un instrumento de medición que los resultados que se obtengan sean reproducibles tanto intraoperador como entre operadores, por ello Brouwers y cols. (25) publicó un estudio con resultados positivos en ese punto, acerca del Ostell Mentor. Debido al coste del Osstell Mentor, Degidi y cols. (26) realizaron un estudio comparativo entre la percepción manual de la estabilidad de los implantes en el momento de la colocación de éstos por un clínico experimentado y la medida real que proporciona el Osstell Mentor, obteniendo diferencias significativas entre los resultados obtenidos entre la estabilidad percibida por el clínico y la obtenida mediante el osstell llegando a la conclusión que por muy experimentado que sea el clínico, esta percepción varía entre individuos, por lo tanto se determinó que el uso del Osstell Mentor reproduce con mayor fiabilidad la estabilidad real del implante.

El diámetro es uno de los parámetros que pueden determinar la estabilidad de los implantes. En los resultados obtenidos en este estudio, al comparar los tres diámetros, se ha encontrado que no hay diferencias estadísticamente significativas, siendo para el implante de menor diámetro el de mayor coeficiente de estabilidad, no coincidiendo con lo publicado por Araceli Bonarat y cols. (27) y Simunek y cols. (10) que sí obtuvieron diferencias estadísticamente significativas en cuanto al diámetro siendo mayor la estabilidad en los implantes de mayor diámetro. Sin embargo autores como Huwiler y cols. (28) tampoco encontraron diferencias significativas entre los diferentes diámetros, al igual que lo publicado por Bischof (29). Con respecto a la longitud, tampoco se han encontrado diferencias estadísticamente significativas, obteniendo el coeficiente de estabilidad mayor para el implante de menor longitud, coincidiendo con los estudios publicados por Mashiko (9), Mihoko y cols. (22) y Payam y cols. (30). De igual manera Araceli Bonarat, en un estudio donde comparaban implantes de diferentes longitudes, no obtuvo diferencias estadísticamente significativas siendo la estabilidad primaria mayor para el implante corto. Por lo contrario, autores como Stephen y cols. (31) y Barewal y cols. (3) obtuvieron menor estabilidad en los implan- 
tes cortos, pero sí coinciden con este estudio al no encontrar diferencias estadísticamente significativas entre las diferentes longitudes, como tampoco encontraron Ersanli (16), Brochu y cols. (32) y Calvo y cols. (33) al estudiar diferentes parámetros, entre ellos la longitud, obtuvieron mayor coeficiente de estabilidad en el implante de $10 \mathrm{~mm}$ que en los implantes de $13 \mathrm{~mm}$, no siendo tampoco el resultado significativo.

\section{CONCLUSIONES}

Tras el estudio realizado se puede concluir:

1. El diámetro no es factor determinante del coeficiente de estabilidad.

2. La longitud no influye en el coeficiente de estabilidad.

3. El utilizar implantes denominados anchos no implica que los resultados de estabilidad sean mayores que en implantes estrechos.

4. A mayor longitud, el coeficiente de estabilidad disminuye.

\section{BIBLIOGRAFÍA}

1. Da Cunha HA, Francischon CE, Filho HN, de Oliveira RC. A Comparison Between Cutting Torque and Resonance Frequency in the Assessment of Primary Stability and Final Torque Capacity of Standard and TiUnite SingleTooth Implants Under Immediate Loading. Int J Oral Maxillofac Implants 2004;19(4):578-85.

2. Şençimen M GA, Özen J, Dergin C, Okçu KM, Ayyildiz S, Altuğ HA. Early Detection of Alterations in the Resonance Frequency Assessment of Oral Implant Stability on Various Bone Types: A Clinical Study. J Oral Implantol 2011;37(4):411-9.

3. Barewal RM, Meredith N, Cochran DL. Resonance frequency measurement of implant stability in vivo on implants with a sandblasted and acid-etched surface. THe Int J Oral Maxillofac Implants 2003;18:641-51.

4. Friberg B, Grondahl K, Lekholm U. A New Self-Tapping Brånemark Implant: Clinical and Radiographic Evaluation. Int J Oral Maxillofac Implants 1992;7:80-5.

5. Aparicio C, Rangert B, Sennerby L. Immediate/early loading of dental implants: A report from the Sociedad Española de Implantes World Congress consensus meeting in Barcelona, Spain 2002. Clin Implant Dent Relat Res 2002;5:57-60.
6. Anitua E, et al. Clinical Outcome of Immediately Loaded Dental Implants Bioactivated With Plasma Rich in Growth Factors: A 5-Year Retrospective Study.J Periodontol 2008; 79(7):1168-76.

7. Cannizzaro G, Leone M, Espósito M. Immediate Functional Loading of Implants Placed with Flapless Surgery in the Edentulous Maxilla: 1-year Follow-up of a Single Cohort Study. Int J Oral Maxillofac Implants 2007;22:8795.

8. Abrahamsson I, Linder E. Lang NP. Implant stability in relation to osseointegration: an experimental study in the Labrador dog. Clin Oral implants Res 2009;20(3): 313-8.

9. Yamaguchi M,Xu H,ShimizuY,Hatano N, Ooya K. Resonance frequency analysis of long-term implant success in the posterior partially edentulous mandible. Quintessence Int 2008;39(3):121-5.

10. Simunek A, Strnad J, Kopecka D, Brazda T, Pilathadka, S Chauhan R, Slezak R. Changes in Stability After Healing of Immediately Loaded Dental Implants. Int J Oral Maxillofac implants 2010;25(6):1085-92.

11. Cehreli MC, Kökat AM, Comert A, Akkocaoglu M, Tekdemir I, Akca K. Implant stability and bone density: assessment of correlation in fresh cadavers using conventional and osteotome implant sockets. Clin Oral Implants Res 2009;20(10):1163-9.

12. Ito Y, Sato D, Yoneda S, Ito D, Kondo H, Kasugai S. Relevance of resonance frequency analysis to evaluate dental implant stability: simulation and histomorphometrical animal experiments. Clin Oral Implants Res 2008; 19(1):9-14.

13. Johansson C, Albrektsson T. Integration of Screw Implants in the Rabbit: A l-yr Follow-up of Removal Torque of Titanium Implants. JOMI 1987: 69-75.

14. Stein AE, McGlmply EA, Johnston WM, Larsen PE. Effects of Implant Design and Surface Roughness on Crestal Bone and Soft Tissue Levels in the Esthetic Zone. Int J Oral Maxillofac Implants 2009;24(5):910-9.

15. Lachmann S, Jäger B. Axmann D, Gómez-Román G, Groten M, Weber H. Resonance frequency analysis and damping capacity assessment. Part I: an in vitro study on measurement reliability and a method of comparison in the determination of primary dental implant stability. Clin Oral Implants Res 2006;17(1):75-9. 
16. Ersanli S, Karabuda C, Beck F, Leblebicioglu B. Resonance Frequency Analysis of One-Stage Dental Implant Stability During theOsseointegration Period. J Periodontol 2005; 76(7):1066-71.

17. Huang HM, Chiu CL, Yeh CY, Lin CT, Lin LH, Lee SY. Early detection of implant healing process using resonance frequency analysis. Clin Oral Res 2003;14:437-43.

18. Lachmann S, Laval JY, Jäger B, Axmann D, Gómez-Román G, Groten M,Weber H. Resonance frequency analysis and damping capacity assessment. Part 2: peri-implant bone loss follow-up. An in vitro study with the Periotest and Osstell instruments. Clin Oral Implants Res 2006;17(1):80-4.

19. Shiigai T. Pilot study in the identification of stability values for determining immediate and early loading of implants. J Oral Implantol 2007;33(1):13-22.

20. Glauser R, Sennerby L, Meredith N, Reé A, Lundgren A, Gottlow J, Hämmerle CH. Resonance frequency analysis of implants subjected to immediate or early functional occlusal loading. Successful vs. failing implants. Clin Oral Implants Res 2004;15(4):428-34.

21. Sennerby L, Meredith N. Implant stability measurements using resonance frequency analysis: biological and biomechanical aspects and clinical implications. Periodontol 2000. 2008;47:51-66.

22. Atsumi M, Park SH, Wang HL. Methods Used to Assess Implant Stability: Current Status. Int J Oral Maxillofac Implants 2007;22(5):743-54.

23. Buser D, Nydegger T, Hirt HP, Cochran DL, Nolte LP. Removal Torque Values of Titanium Implants in the Maxilla of Miniature Pigs. Int J Oral Maxillofac Implants 1998;13 (5):611-9.

24. Cornelini R, Cangini F, Covani U, Barone A, Buser D. Immediate Restoration of Single-Tooth Implants in Mandibular Molar Sites:A 12-month Preliminary Report. Int J Oral Maxillofac Implants 2004;19(6):855-60.

25. Brouwers JE, Lobbezoo F, Visscher CM, Wismeijer D, Naeije M. Reliability and validity of the instrumental assessment of implant stability in dry human mandibles. J Oral Rehabil 2009;36(4):279-83.

26. Degidi M, Daprile G, Piattelli A. Determination of Primary Stability: A Comparison of the Surgeon's Perception and
Objective Measurements. Int J Oral Maxillofac Implants 2010;25:558-61.

27. Boronat-López A, Peñarrocha-Diago M, MartínezCortissoz O, Mínguez-Martínez I. Estudio del análisis de frecuencia de resonancia tras la colocación de 133 implantes dentales. Med Oral Patol Oral Cir Bucal 2006; 11:272-6.

28. Huwiler MA, Pjetursson BE, Bosshardt DD, Salvi GE, Lang NP. Resonance frequency analysis in relation to jawbone characteristics and during early healing of implant installation. Clin Oral Implants Res 2007;18(3):275-80.

29. Bischof M, Nedir R, Szmukler-Moncler S, Bernard JP, Samson J. Implant stability measurement of delayed and immediately loaded implants during healing. Clin Oral Implants Res 2004;15(5):529-39.

30. Payam F, Andersson L, Sten G, Sharma O. Implant Stability, Tissue Conditions, and Patient Self-Evaluation after Treatment with Osseointegrated Implants in the Posterior Mandible. Clin Implant Dent Relat Res 2004;6(1):2432.

31. Balshi SF, Allen D, Wolfinger GJ, Balshi TJ. A Resonance Frequency Analysis Assessment of Maxillary and Mandibular Immediately Loaded Implants. Int J Oral Maxillofac Implants 2005;20:584-94.

32. Brochu JF, Anderson JD, Zarb GA. The Influence of Early Loading on Bony Crest Height and Stability: A Pilot Study. Int J Prosthodont 2005;18:506-12.

33. Calvo Guirado JL, Ortiz Ruíz J, Gómez Moreno G, López Marí L, Bravo González LA. Immediate loading and immediate restoration in 105 expanded-platform implants via the Diem System after a 16-month followup period. Med Oral Patol Oral Cir Bucal 2008;sepl;13 (9):576-81.

\section{CORRESPONDENCIA}

\section{N. Cervantes Haro}

Instituto Mississippi

Departamento de Cirugía

Santísima Trinidad, 5

28010 Madrid

E. mail contacto: saraycalpe@gmail.com 